DOI:

УДК 62-34

ИССЛЕДОВАНИЕ АВТОМАТИЗИРОВАННОГО ПРИВОДА УПРАВЛЕНИЯ РУЧНЫМИ ГИДРОРАСПРЕДЕЛИТЕЛЯМИ МАНИПУЛЯТОРА

кандидат технических наук, доцент Л. Д. Бухтояров ${ }^{1}$

кандидат технических наук М. Н. Лысыч ${ }^{1}$

кандидат медицинских наук, доцент Я. А. Туровский ${ }^{2}$

1 - ФГБОУ ВО «Воронежский государственный лесотехнический университет имени Г.Ф. Морозова»,

г. Воронеж, Российская Федерация

2 - ФГБОУ ВО «Воронежский государственный университет», г. Воронеж, Российская Федерация

Статья посвящена проблемамавтоматизациисистем управлениямашинами, имеющими гидравлический привод. Ключевым управляющим компонентом гидросистем стационарных машин, станков, прессов, тракторов, автокранов, экскаваторов, сельскохозяйственных машинявляются гидрораспределители используемые для изменения направления или пуска и остановки рабочей жидкости. На сегодняшний день наряду с гидрораспределителями имеющими ручное управление все более широкое распространение находят распределители созданные на основе сервоприводов, шаговых двигателейи с электромагнитным управлением. Это позволяют достигнуть высокой точности управления потоками жидкости и максимально автоматизировать процессы управления за счет возможности непосредственной коммутации привода с управляющими компьютерными системами.Авторами предлагается конструкция автоматизированного привода на основе шаговых двигателей которыйустанавливаетсянепосредственно на гидрораспределитель с ручным управлением. Подобный подход исключает переделку самой гидросистемы, что значительно снижает затраты и трудоемкость процесса модернизации. При проектировании привода была использована среда автоматизированного проектирования SolidWorks. B рамках теоретических исследований проведены кинематические и динамических исследованияпроцесса управления гидрораспределителем. Для этого использовались расчетные 3D-модели привода с гидрораспределителем и приложение для инженерных расчетов SolidWorksSimulation. В эксперименте моделировались три режима работы привода: с постоянной скоростью; с переменной скоростью; режим пульсации. Проведенанализданныхмоделирования.Для практического подтверждения работоспособности предлагаемой конструкции был изготовлен макетный образец привода, который устанавливался на гидроманипулятор и создана управляющая компьютерная программа. Проведенные экспериментальные исследования по управлению гидрораспределителем манипулятора показали достаточно хорошую управляемость и точность позиционирования.

Ключевые слова: гидрораспределитель, автоматизированный привод, САПР, имитационное моделирование, экспериментальные исследования

\title{
RESEARCH OF THE AUTOMATED DRIVE OF MANUAL HYDRAULIC VALVES OF THE MANIPULATOR
}

$\mathrm{PhD}$ of Engineering, Associate Professor L. D. Bukhtoyarov ${ }^{1}$

$\mathrm{PhD}$ of Engineering, Senior Lecturer M. N. Lysych ${ }^{1}$

$\mathrm{PhD}$ of Biology, Associate Professor Y. A. Turovskiy ${ }^{2}$

1 - Federal State Budget Education Institution of Higher Education «Voronezh State University of Forestry and Technologies named after G.F. Morozov», Voronezh, Russian Federation

2 - Federal State Budget Education Institution of Higher Education «Voronezh State University»,

Voronezh, Russian Federation

\begin{abstract}
Article is devoted to problems of automation of control systems by the machines having the hydraulic drive. The key controlling component of hydraulic systems of stationary machines, machines, presses, tractors, truck cranes, excavators, Today along with the hydraulic valves having manual control, distributors, created on the basis of servo actuators, stepping motors and
\end{abstract}




\section{Технологии. Машины и оборудование}

with electromagnetic control, become more and more popular. It allow to reach high accuracy of control of liquid flows and as much as possible to automate control processes at the expense of a possibility of direct switching of the drive with the controlling computer systems. Authors offer a design of the automated drive on the basis of step engines which is mounted directly on the hand-operated hydraulic valve. Similar approach excludes alteration of the hydraulic system that considerably reduces costs and labor input of process of upgrade. When designing the drive the SolidWorks environment of the automated designing was used. In the experiment three operation modes of the drive were modeled: with constant speed; with variable speed; pulsation mode. Simulation data analysis is carried out. For practical confirmation of operability of the offered construction the model sample of the drive which was set on a hydraulic manipulator was made and the controlling computer program is created. The conducted pilot studies on control of the hydraulic valve of the pointing device showed rather good controllability and positioning accuracy.

Keywords: hydraulic distributor, automated drive, CAD, simulation, experimental studies

Гидрораспределители применяется для изменения направления или пуска и остановки рабочей жидкости в гидравлических системах стационарных машин, станков, прессов, гидросистем тракторов, автокранов, экскаваторов, сельскохозяйственных машин и т.д.

По управлениюгидрораспределители подразделяются на гидроаппараты с ручным, электромагнитным, пневматическим, гидравлическим или электрогидравлическим управлением $[1,4]$.

Большой интерес представляют системы управления гидрораспределителями созданные на основе сервоприводов и шаговых двигателей. Подобные приводы позволяют достигнуть высокой точности управления потоками жидкости и максимально автоматизировать процессы управления. Это достигается за счет возможности непосредственной коммутации привода с управляющими компьютерами $[5,6,7,8,9,10,11,12,13]$.

Однако на данный момент существует огромное количество машин, оснащенных исключительно гидрораспределителямис ручным приводом, а это далеко не всегда позволяет обеспечить их наиболее эффективное применение. Один из способов повышения эффективности подобных машин это создание автоматизированных приводов, которые позволяют, без какой-либо переделки конструкции распределителя максимально автоматизировать его работу. Реализация подобного подхода должна повысить производительность и качество работы гидроагрегатов. При этом стоимость подобных систем должна быть минимальной.

Для решения поставленной задачи была предложена конструкция автоматизированного привода и концепция управления им. Для обоснования конструк- ции привода была создана его 3D модель в среде SolidWorks.

На рис. 1 представлен общий вид автоматического привода, разработанного для гидрораспределителя НС-M50.

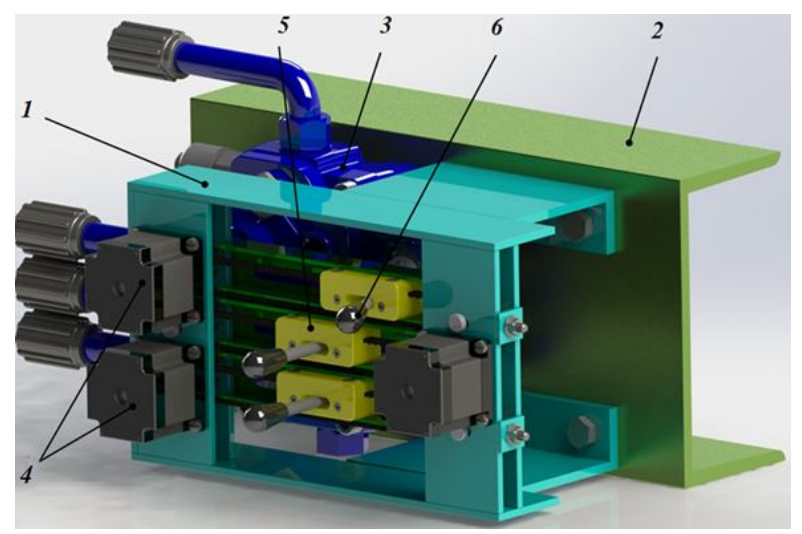

Рис. 1. Общий вид автоматического привода гидрораспределителя НC-M50

Привод состоит из рамы 1 жестко закрепленной на основании 2 к которому крепится гидрораспределитель 3 , шаговые электродвигатели 4 , и непосредственно механизм перемещения 5 рычагов управления 6.

Механизм перемещения состоит из зубчатых колес 1 , зубчатого ремня 2, разъемных кареток $3 \mathrm{c}$ металлическим вкладышем, механизмов натяжения зубчатых ремней 4 и направляющих 5 (рис. 2) [2].

Привод работает следующим образом. Управляющие команды, формируемые оператором или компьютерной программой, передаются через блоки управления шаговым двигателям. Посредством ременной зубчатой передачи вращательное движение вала двигателя преобразуется в поступательное движение каретки, которая в свою очередь, отклоняет ручки 


\section{Технологии. Машины и оборудование}

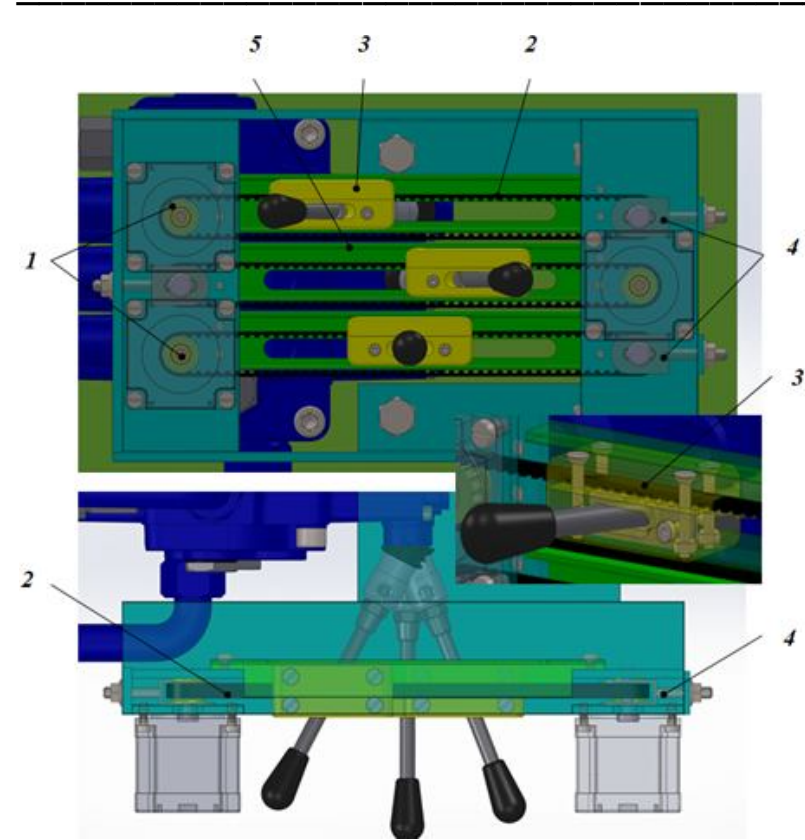

Рис. 2. Механизм перемещения рычагов гидрораспределителя НС-M50

управления гидрораспределителя.

Преимуществом подобной схемы следует отнести высокую скорость работы привода и возможность быстрого возврата ручки распределителя в нейтральное положение за счет его пружины. При использовании самотормозящихся передач типа винт-гайка это было бы невозможным, что могло бы вести к утере нейтрального положения и необходимости регулярных наладок. К недостаткам примененной схемы следует отнести потребность в шаговых двигателях достаточно большой мощности.

На базе 3D-модели также была разработана расчетная имитационная модель в приложении SolidWorks Simulation [3].

При моделировании задавались следующие параметры:

- удержание и возврат в нейтральное положение рычага распределителя осуществляется виртуальной пружиной с линейным законом изменения жесткости и коэффициентом упругости 2,00 Н/мм;

- 3D контакт в месте взаимодействия ручки распределителя и стального вкладыша каретки - сухой контакт сталь-сталь;

- материалы подвижных частей привода: АБС пластик для каретки и наконечника ручки, углеродистая сталь для ручки, винтов, гаек и вкладыша;

- перемещение кареток осуществлялось приложением линейных двигателей к их торцевой поверхности. Задание скоростных параметров осуществлялось с помощью интегрированного конструктора функций (рис. 3).

При моделировании воспроизводились три различных режима работы: перемещение с постоянной

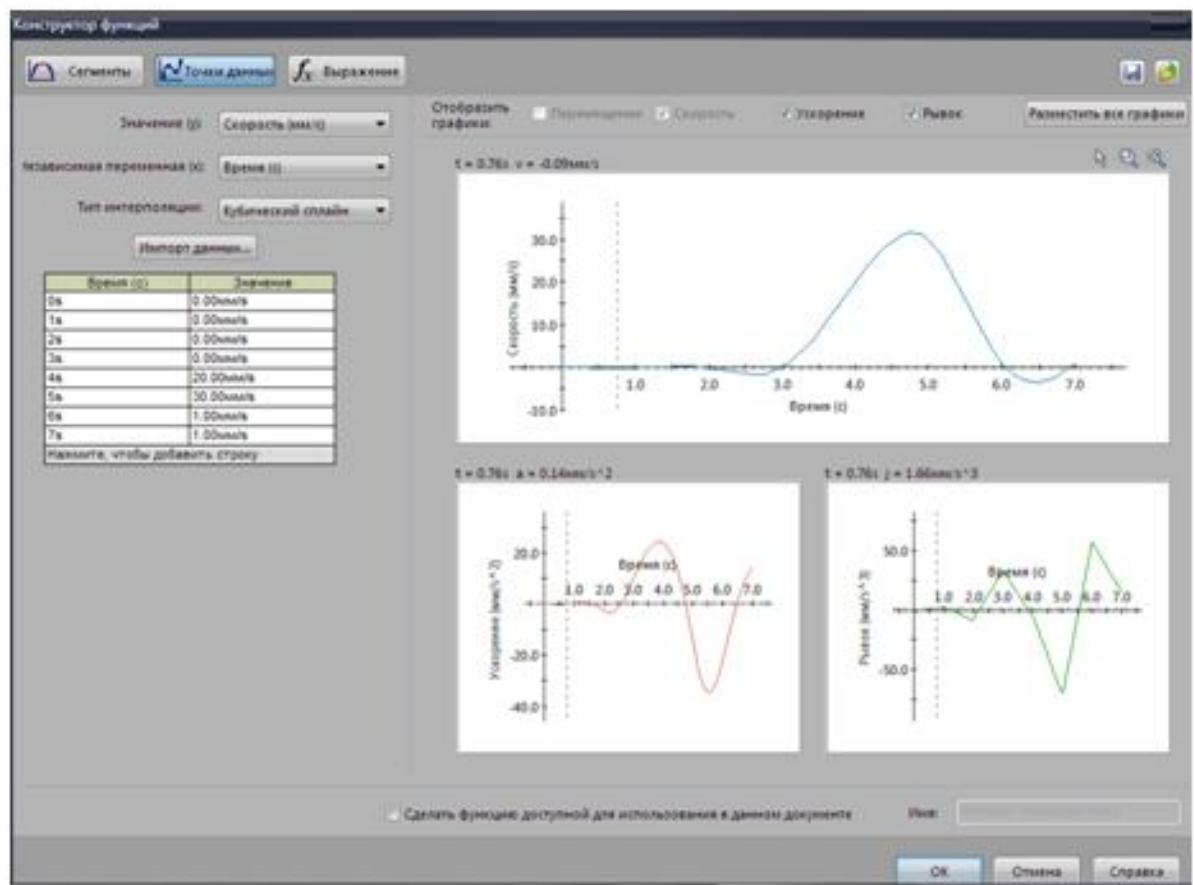

Рис. 3. Задание закона движения каретки привода 


\section{Технологии. Машины и оборудование}

скоростью (рис. 4, a); с переменной скоростью (рис. 4, б); режим пульсации (рис. 4, в).

В процессе моделирования производилось отслеживание следующих параметров: линейные перемещения кареток, угловые перемещения ручек распределителя, угловые скорости и ускорения, силы на виртуальных линейных двигателях и силы сопротивления пружин. Непосредственно на экране отслеживались линейные перемещения кареток привода и усилия на виртуальных двигателях (рис. 4).

Полученные в результате моделирования данные (рис. 5) позволяют оценить быстродействие привода и его силовуюнагруженность. Это необходимо для выбора оптимальных параметров привода и установления допустимых режимов работы. Возможность отслеживания угловых перемещений открывает возможности для расчета расхода жидкости и соответствующих перемещений исполнительных органов. Модель также имеет возможность ввода обратных силовых воздействий со стороны гидросистемы на ручки управления, которые могут быть получены экспериментальным путем.

На основе разработанной 3D модели был изготовлен экспериментальный образец автоматизированного привода. Он был установлен на гидрораспределитель манипулятора (рис. 6). На приведенной фотографии видно, что штатное место гидрораспределителя находится непосредственно под стрелой, что ухудшает обзор и, следовательно, управляемость манипулятора, а самое главное - не обеспечивает безопасность оператора.

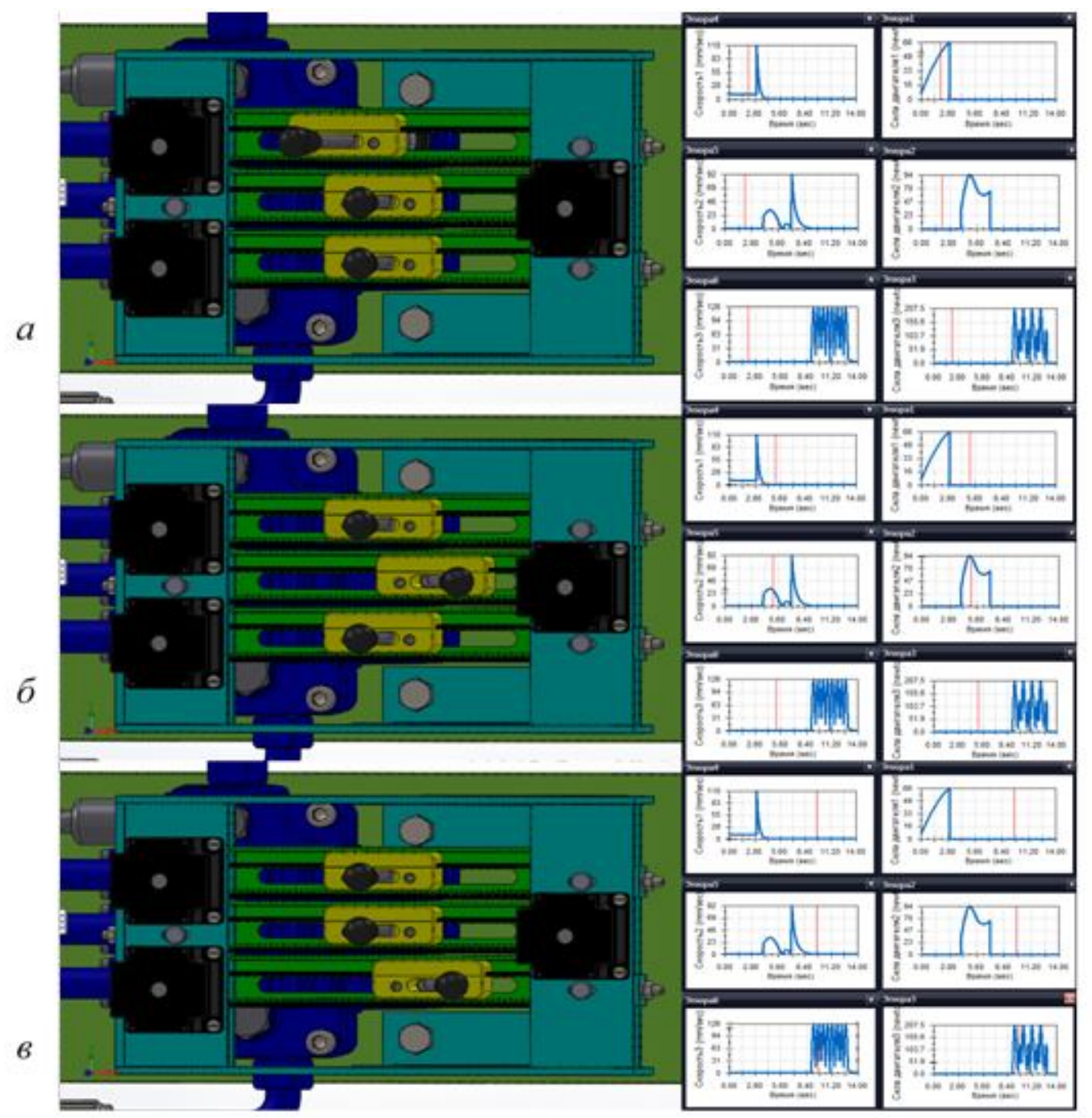

Рис. 4. Процесс моделирования работы привода с фиксированием динамических силовых характеристик: $a$ - перемещение с постоянной скоростью; $\sigma$ - с переменной скоростью; $в$ - режим пульсации 

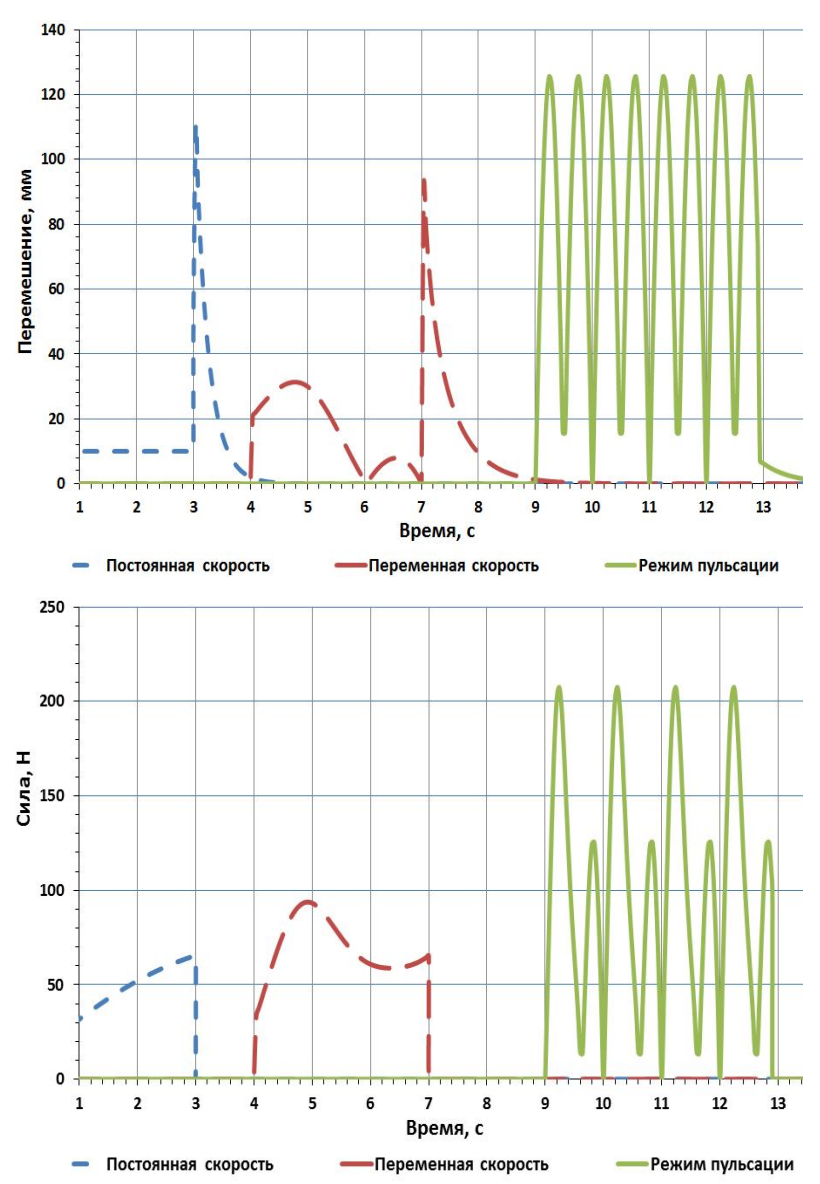

Рис. 5. Результаты моделирования работы привода $a$ - скорость перемещения каретки в зависимости от режимов испытания; $\sigma$ - сила, требуемая для перемещения каретки

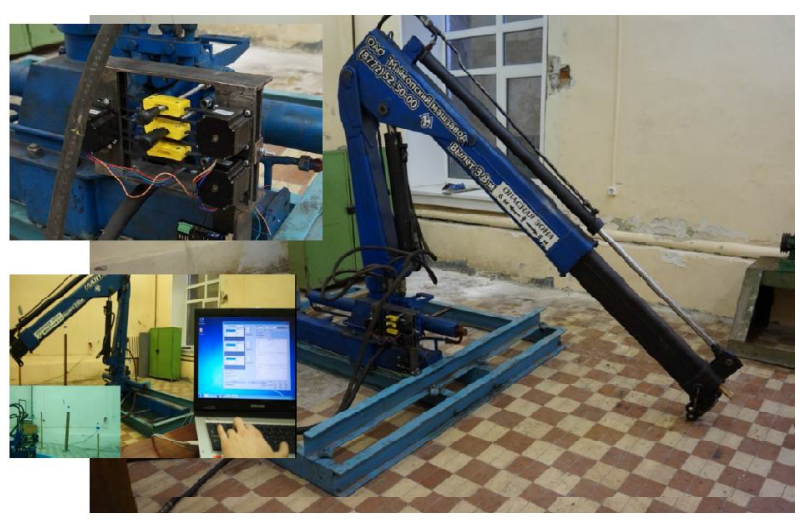

Рис. 6. Экспериментальный образец автоматизированного привода установленный на манипулятор

Управление приводом осушествляется посредством созданной нами программы для ЭВМ, которая подаёт команды блокам управления шаговыми двигателями. На рис. 7 представлен ее интерфейс.

Программа может управлять несколькими блоками управления, но для наглядности представлена работа с одним блоком управления.

Перед началом работы требуется построить график скоростей двигателя рабочего интервала времени. Для этого требуется задать значения времени и соответствующих им скоростей для нескольких ключевых моментов, а также шаг времени в секундах для требуемой точности значений графика. Для плавности изменения скоростей шаговых двигателей используется линейная интерполяция или построение кубического сплайна на основе ключевых значений, заданных в

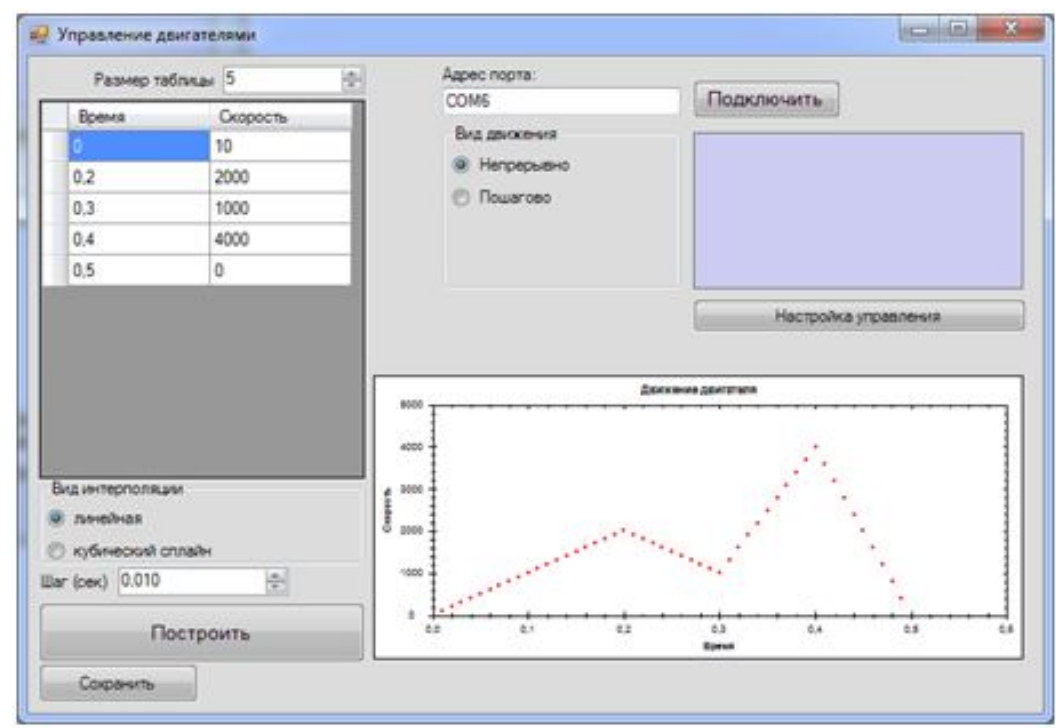

Рис. 7. Интерфейс программы управления шаговыми двигателями 


\section{Технологии. Машины и оборудование}

таблице. Затем происходит подключение блока управления шаговых двигателей пугём ввода адреса порта и нажатия кнопки «Подключить». После этого выбирается требуемый вид движения: непрерывный или пошаговый. Для подачи команд управления требуется установить курсор в области ввода, а затем нажимать на клавиши клавиатуры, которые соответствуют различным режимам движения шаговых двигателей. При этом пользователю даётся возможность настраивать кнопки управления по собственному желанию.

Вывод. Применение подобных приводов способно повысить производительность, качество и безопасность выполняемых операций. При этом капитальные вложения в совершенствование оборудования сравнительно невысоки.

\section{Библографический список}

1. Гидроманипуляторы и лесное технологическое оборудование [Текст] : монография / И. М. Бартенев, 3. К. Емтыль, А. П. Татаренко, М. В. Драпалюк, П. И. Попиков ; под ред. И. М. Бартенева. - М. : ФЛИНТА, Наука, 2011. - 408 с.

2. Бухтояров Л.Д. Автоматизированный привод для управления ручными гидрораспределителями [Текст] / Л.Д. Бухтояров, М.Н. Лысыч, Р.В. Мирзеханов // Актуальные направления научных исследований XXI века: теория и практика : сборник научных трудов по материалам международной заочной научно-практической конференции. - Воронеж, 2015. - № 7, ч. 1 (18-1). - С. 187-190.

3. Бухтояров Л.Д. Моделирование работы автоматизированного привода для гидрораспределителя с ручным управлением [Текст] / Л.Д. Бухтояров, М.Н. Лысыч, Р.В. Мирзеханов // Актуальные направления научных исследований XXI века: теория и практика : сборник научных трудов по материалам международной заочной научно-практической конференции. - Воронеж, 2015. - № 5, ч. 4 (16-4). - С. 51-54.

4. Панченко А.И.Основные направления гидрофикации мобильной техники [Текст] / А.И.Панченко, А.А.Волошина, Ю.П. Обернихин // ПраціТаврійського державного агротехнологічногоуніверситету. - 2013. № 13. - T. 6. - C. 3-19.

5. Amirante, R. Evaluation of the flow forces on a direct (single stage) proportional valve by means of a computational fluid dynamic analysis [Text] / R. Amirante, P.G. Moscatelli, L.A. Catalano // Energy Conversion and Management. - 2007. - no. 3. - pp. 942-953.

6. Baghestan, K. An energy-saving nonlinear position control strategy for electro-hydraulic servo systems [Text] / K. Baghestan, S. Rezaei, H. Talebi, M. Zareinejad // ISA Transactions. - 2015. - pp. 268-279.

7. Bonchis, A. Variable structure methods in hydraulic servo systems control [Text] / A. Bonchis, P. Corke, D. Rye, Q. Ha // Automatica. 2001. - no. 4 (37). - pp. 589-595.

8. Chen, C. An electro-hydraulic servo control system research for CFETR blanket RH [Text] / C. Chen, H. Tang, S. Qi, Y. Cheng, H. Feng, X. Peng, Y. Song // Fusion Engineering and Design. - 2014. - no. 11. - pp. 2806-2813.

9. Keles, O. Theoretical and experimental investigation of a pulse-width modulated digital hydraulic position control system[Text] / O. Keles, Y. Ercan // Control Engineering Practice. - 2002. - no. 6. - pp. 645-654.

10. Lee, J. Design and experimental evaluation of a robust position controller for an electrohydrostatic actuator using adaptive antiwindup sliding mode scheme $[$ Text] / J. Lee, S. Park, J. Kim, J. Lee, S. Park, J. Kim // The Scientific World Journal. - 2013. - pp. 1-16.

11. De Negri V. Behavioural prediction of hydraulic step-up switching converters [Text] / De Negri V., P. Wang, A. Plummer, D. Johnston // International Journal of Fluid Power. - 2014. - no. 1 (15). - pp. 1-9.

12. Ruan, J. Domain Modelling and Identification of 2D Digital Servo Valve [Text] / J. Ruan, P.R. Ukrainetz, R. Burton // International Journal of Fluid Power. - 2000. - no. 2. - pp. 49-59.

13. Sirouspour, M.R. On the nonlinear control of hydraulic servo-systems [Text] / M.R. Sirouspour, S.E. Salcudean // Robotics and Automation, - 2000. - Vol. 2. - pp. 1276-1282.

14. Takahashi, T. Development of water hydraulic proportional control valve [Text] / T. Takahashi, P. Yamashina, S. Miyakawa // Proceedings of the JFPS International Symposium on Fluid Power. - 1999. - no. 4. - pp. 549-554. 


\section{References}

1. Bartenev I.M., Emtyl Z.K., Tatarenko A.P., Drapaljuk M.V., Popikov P.I. Gidromanipulyatory i lesnoe tekhnologicheskoe oborudovanie [Hydromanipulators and the wood process equipment].Moscow, 2011,408 p. (in Russian).

2. Bukhtoyarov L.D., Lysych M.N., Mirzekhanov R.V. Avtomatizirovannyj privod dlja upravlenija ruchnymi gidroraspredeliteljami [The automated drive for management of manual hydrodistributors] Aktual'nye napravlenija nauchnyh issledovanij XXI veka: teorija i praktika. [Actual Directions of Scientific Researches of the XXI-st century: the Theory And Practice]. Voronezh, 2015, no. 7-1 (18-1) (3), pp. 187-190 (in Russian).

3. Bukhtoyarov L.D., Lysych M. N, Mirzekhanov R.V. Modelirovanie raboty avtomatizirovannogo privoda dlja gidroraspredelitelja s ruchnym upravleniem [Simulation of the automated drive for manual hydraulic distributor] $A k$ tual'nye napravlenija nauchny issledovanij XXI veka: teorija i praktika. [Actual Directions of Scientific Researches of the XXI-st century: the Theory And Practice]. Voronezh, 2015, no. 5-4 (16-4) (3), pp. 51-54(in Russian).

4. Panchenko A.I., Voloshina A.A., ObernikhinYu.P. Osnovnye napravlenija gidrofikacii mobil'noj tehniki [The basic directions hydraulic the mobile technics] PraciTavrijs'kogo derzhavnogo agrotehnologichnogouniversitetu [Works of Tavrijsky state agrotechnical university]. 2013, no. 13, Vol. 6, pp. 3-19 (inRussian).

5. Amirante R., Moscatelli P.G., Catalano L.A. Evaluation of the flow forces on a direct (single stage) proportional valve by means of a computational fluid dynamic analysis. Energy Conversion and Management. 2007, no. 3, pp. 942-953.

6. Baghestan K., Rezaei S., Talebi H., Zareinejad M. An energy-saving nonlinear position control strategy for electro-hydraulic servo systems. ISA Transactions. 2015, pp. 268-279.

7. Bonchis A.,Corke P., Rye D., Ha Q. Variable structure methods in hydraulic servo systems control. Automatica. 2001, no. 4 (37), pp. 589-595.

8. Chen C.,Tang H., Qi S., Cheng Y., Feng H., Peng X., Song Y. An electro-hydraulic servo control system research for CFETR blanket RH. Fusion Engineering and Design. 2014, no. 11, pp. 2806-2813.

9. Keles O., Ercan Y. Theoretical and experimental investigation of a pulse-width modulated digital hydraulic position control system. Control Engineering Practice, 2002, no. 6, pp. 645-654.

10. Lee J., Park S., Kim J., Lee J., Park S., Kim J. Design and experimental evaluation of a robust position controller for an electrohydrostatic actuator using adaptive antiwindup sliding mode scheme. The Scientific World Journal. 2013, pp. 1-16.

11. De Negri V., Wang P., Plummer A., Johnston D. Behavioural prediction of hydraulic step-up switching converters. International Journal of Fluid Power. 2014, no. 1 (15), pp. 1-9.

12. Ruan J., Ukrainetz P.R., Burton R. Frequency. Domain Modelling and Identification of 2D Digital Servo Valve. International Journal of Fluid Power. 2000, no. 2, pp. 49-59.

13. Sirouspour M.R., Salcudean S.E. On the nonlinear control of hydraulic servo-systems. Robotics and Automation, 2000. Proceedings. ICRA '00. IEEE International Conference on. 2000, Vol. 2, pp.1276-1282.

14. Takahashi T., Yamashina P., Miyakawa S. Development of water hydraulic proportional control valve. Proceedings of the JFPS International Symposium on Fluid Power. 1999, no. 4, pp. 549-554.

\section{Сведения об авторах}

Бухтояров Леонид Дмитриевич - заведующий кафедры лесной промышленности, метрологии, стандартизации и сертификации ФГБОУ ВО «Воронежский государственный лесотехнический университет имени Г.Ф. Морозова», кандидат технических наук, доцент, г. Воронеж, Российской Федерации; e-mail: vglta-mlx@yandex.ru

Лыссыч Михаил Николаевич - старший преподаватель кафедры лесной промышленности, метрологии, стандартизации и сертификации ФГБОУ ВО «Воронежский государственный лесотехнический университет имени Г.Ф. Морозова», кандидат технических наук, г. Воронеж, Российской Федерации; e-mail: miklynea@yandex.ru

Туровский Ярослав Александрович - заведующий лабораторией медицинской кибернетики ФГБОУ ВО «Воронежский государственный университет», кандидат медицинских наук, доцент, г. Воронеж, Российской Федерации; e-mail: yaroslav_turovsk@mail.ru 


\section{Технологии. Машины и оборудование}

\section{Information about authors}

Bukhtoyarov Leonid Dmitrievich - Head of Department of Forest Industry, Metrology, Standardization and Certification department, Federal State Budget Education Institution of Higher Education «Voronezh State University of Forestry and Technologies named after G.F. Morozov», Фssociate Professor, Ph.D. in Engineering, Voronezh, Russian Federation; e-mail: vglta-mlx@yandex.ru

Lysych Mikhail Nikolaevich - Senior Lecturer of Forest Industry, Metrology, Standardization and Certification department, Federal State Budget Education Institution of Higher Education «Voronezh State University of Forestry and Technologies named after G.F. Morozov»», Ph.D. in Engineering, Voronezh, Russian Federation; e-mail: miklynea@yandex.ru

TurovskiyYaroslavAleksandrovich - Head of the laboratory of Medical Cybernetics, Federal State Budget Education Institution of Higher Education «Voronezh State University», PhD of Biological, Associate Professor, Voronezh, Russian Federation; e-mail: yaroslav_turovsk@mail.ru

DOI:

УДК $630 * 377.44$

К ОПРЕДЕЛЕНИЮ БУКСОВАНИЯ ГУСЕНИЧНОЙ ЛЕСОЗАГОТОВИТЕЛЬНОЙ МАШИНЫ

кандидат технических наук, доцент Е. Е.Клубничкин ${ }^{1}$

кандидат технических наук, доцент В. Е.Клубничкин ${ }^{1}$

доктор технических наук, профессор Г. О. Котиев ${ }^{2}$

доктор технических наук, профессор О. А. Наказной ${ }^{2}$

1 - Мытищинский филиал ФГБОУ ВО «Московский государственный технический университет им Н.Э. Баумана», г. Мытищи, Российская Федерация

2 - ФГБОУ ВО «Московский государственный технический университет им Н.Э. Баумана»

г. Москва, Российская Федерация

\section{Работа выполнена при поддержке Министерства образования и науки Российской Федерации проект № 1547}

Повышение энергонасыщенности и развитие технологической навески лесопромышленных машин требует оценки влияния скорости и массы машины на буксование движителя, которое в значительной мере определяет проходимость на переувлажнённых грунтах. Для гусеничных лесозаготовительных машин наибольшие сопротивления наблюдаются на грунтах с низкой несущей способностью. Буксование гусеничной лесозаготовительной машины оказывает значительное влияние на её тяговую характеристику и тем самым на энергетический баланс, энергетический потенциал производительности и непосредственно на производительность самой лесозаготовительной машины. В отличии от гусеничного сельскохозяйственного трактора, для которого средний коэффициент буксования в процессе рабочего элемента цикла составляет 2 - 10\%, для лесопромышленного трактора он составляет 10 - 30\% при значительной вероятности работы более 30\%. Исследовали буксование лесозаготовительной машины при постоянных и случайных сцепом весе и тяговой нагрузке. При этом параметры распределения вероятностей практически не зависят от массы лесозаготовительной машины, типа её ходовой системы, типа трансмиссии (при условии обеспечении тягового усилия "по сцеплению"), вида грунта и обусловливаются стилем работы оператора лесозаготовительной машины, в определённой мере, конструкции и параметрами системы управления технологическим оборудованием. При проведении исследований получены кривые изменения буксования движителя лесозаготовительной машины Л3-5 в порожнем и груженом состоянии при различных скоростях движения с учетом буксования холостого хода машины, а также при пренебрежении буксованием холостого хода машины, которые позволили определить оптимум скоростей движения гусеничной лесозаготовительной машин с наименьшим буксованием.

Ключевые слова: гусеничный движитель; лесопромышленная гусеничная машина; движение; ходовая система; нагрузка на грунт. 